\title{
THE SGR A EAST HII COMPLEX AND ASSOCIATED FEATURES
}

\author{
KEVEN I. UCHIDA \\ The Ohio State University \\ MARK R. MORRIS \\ University of California at Los Angeles \\ GENE SERABYN \\ California Institute of Technology \\ DAVID FONG \\ University of Illinois at Urbana Champaigne \\ AND \\ THOMAS MESEROLL \\ Hughes Aircraft Corporation
}

\section{Introduction}

The Sgr A East $\mathrm{H}$ II complex consists of 4 compact $\mathrm{H}$ II regions situated just east of and following, in an arc pattern, the edge of the Sgr A East nonthermal shell. Located between the arc of H II regions and the nonthermal shell is a dense molecular ridge - presumably compressed - known as the " 50 $\mathrm{km} / \mathrm{s}$ cloud". The hypothesis that these $\mathrm{H}$ II regions delineate massive star formation provoked by the rapid expansion of Sgr A East into the molecular cloud is problematical because of the mismatch of the shell expansion and star formation time scales. We therefore examine the alternative hypothesis that Sgr A East is a quasi-static or slowly expanding structure fed from within by the release of relativistic particles from sources at or near the nucleus. The elongation of SgrA East along the Galactic plane is ascribed to the shear inherent in the velocity field this close to the Galactic center (GC). In this proceeding we discuss our ongoing efforts to model the effects of shear in detail, using the elongation of Sgr A East to constrain its expansion time scale. 


\section{Results}

The 2-D kinematic shear modeling was performed with a particle tracing code based on the formulation by Palous \& Tenorio-Tagle (1987) (see also Uchida et al. 1992). Displayed in Figure 1 are the equatorial elements of an expanding GC bubble, as viewed from a position north above the rotational pole of the Galaxy (indicated by the cross); Galactic rotation is in the clockwise sense. The model runs show that in the GC region bubble distortion by shear is inescapable and that for most initial conditions the effects of shear are apparent on relatively short timescales of a few tens of thousands of years. The longitudinal elongation of the Sgr A East nonthermal shell is not at all puzzling in light of the strong shearing present in the GC region.

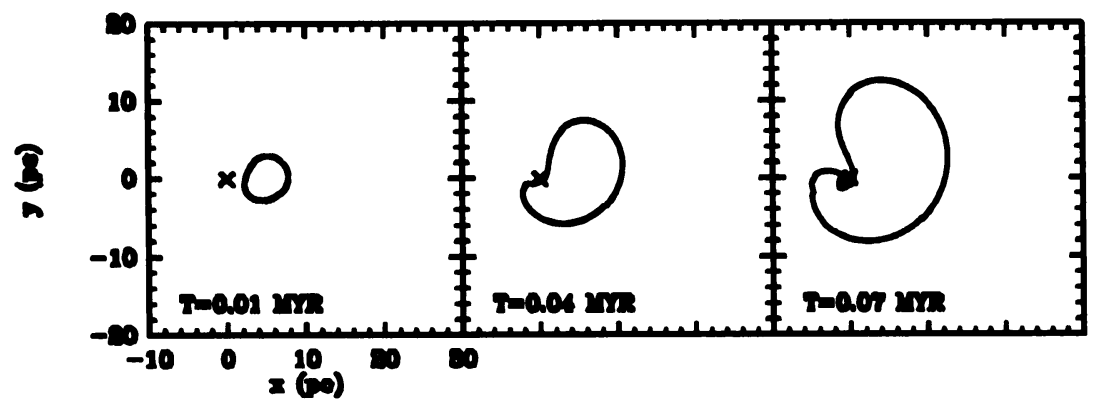

Experimentation with the 2-D code has brought to our attention another interesting phenomenon - the possibility of the shear induced deposition of mass onto the Galactic center by expanding bubbles or arc features. Taken to the extremes in the timesequence, the expanding bubbles wrap around the Galactic center. The tendency of the bubbles to wrap around, rather than to expand past the rotational axis is observed for all but the most extreme of shell expansion velocities explored. A cusp eventually forms in the bubble, where gas presumably shocks and then spirals in to the Galacti: center because of the angular momentum loss it suffers.

We are currently developing a 3-D code which is better adapted to the conditions in the GC region and which will allow us the crucial viewing pespective from within the Galactic plane. We will present this, together with the results from combined interferometer/single-dish molecular line observations of the Sgr A East H II complex, in a future paper.

\section{References}

Tenorio-Tagle G. \& Palous J. 1987, A\&A 186, 287

Uchida, K.I., Morris, M., Bally, J., Pound, M., \& Yusef-Zadeh, F. 1992, ApJ, 398, 128 